\title{
Influence of Solvent Exchange on the Physical Properties of Sodium Silicate Based Aerogel Prepared at Ambient Pressure
}

\author{
Pradip B. Sarawade* Jong-Kil Kim, Jin-Koo Park and Ho-Kun Kim \\ Department of Applied Chemistry, Hanyang University 2-304, Science and Technology Building 1, 1271 \\ Sa 1 Dong, Ansan, Kyunggi-do, 425-791, South Korea.
}

\begin{abstract}
Transparent nanoporous silica aerogel was synthesized at ambient pressure using sodium silicate (water glass) precursor in a sol-gel process. Effect of solvents used for exchange on physical properties was investigated. Trimethylchlorolsilane (TMCS) was used as a hydrophobic reagent in the surface modification process. The volume \% of TMCS was varied by changing its volume from 2 to $12 \mathrm{ml}$ added in $50 \mathrm{ml}$ of hexane and toluene solvent. Also the effects $\mathrm{pH}$ and temperature on the gelation of hydrosol and the physical properties of the nanoporous silica aerogels (particle size, pore volume, surface area, porosity, and pore diameter) were investigated. The thermal stability of the nanoporous silica aerogel was studied in the temperature range 25 to $800^{\circ} \mathrm{C}$. The optical transmittance of the aerogel was found to decrease from 20 to $80 \%$ with an increasing volume $\%$ of TMCS in solvents. The surface chemical modification was confirmed by the FTIR spectroscopy. The modified aerogels were thermally stable up to $350^{\circ} \mathrm{C}$.
\end{abstract}

Keywords: nanoporous silica, sodium silicate, hydrophobicity, BET, PSD, FTIR.

\footnotetext{
${ }^{*}$ Corresponding author. Tel: 82-031-500-3578; Fax: 82-031-500-3578

E-mail address: pradipsarawade@yahoo.co.in
} 


\section{INTRODUCTION}

Silica aerogels are advanced materials developed in the field of materials science. They are highly transparent ( 90\%) in the visible region and highly porous (porosity $~ 98 \%$ ), and with a low refractive index ( 1.01). In addition, they have very low bulk density $\left(\sim 0.030 \mathrm{~g} / \mathrm{cm}^{3}\right)$, low dielectric constant $(\sim 1.7)$, low thermal conductivity ( $\sim 02 \mathrm{~W} / \mathrm{mk})$, low sound velocity $(100 \mathrm{~m} / \mathrm{s})$, and high specific surface area $\left(1,100 \mathrm{~m}^{2} / \mathrm{g}\right)$. They have several technological applications in various fields, such as: inertial confinement fusion targets for thermonuclear fusion reactions, Cerenkov radiation detectors in high energy physics, radio luminescent devices, thermal super insulators in solar energy systems, refrigerators, windows and air-conditioned systems, containers for micrometeorites and liquid rocket propellants, acoustic impendence devices, filters for the automobile exhaust systems and industrial pollutants, and heterogeneous catalysts in various chemical reactions. The preparation of silica aerogels has been extensively studied over the years (Hrubesh, 1988; Pajonk and Teicher, 1985; Svendsen, 1992; Ashley et al., 1992; Pajnok, 1991; Chunha et al., 2000; Deng et al., 1998; Schmidt and Schwertfeger, 1998; Prakash et al., 1995; Yang et al., 1997; Haranath et al., 1997; Schwertfeger et al., 1998). The commercial production of silica aerogels has been limited due to two main reasons: 1) the use of very expensive autoclave for the supercritical drying process; 2) The use of high-cost hazardous alkoxides [TEOS or TMOS] in the sol-gel synthesis. These alkoxides are very expensive, while the supercritical drying process involves the heating and evacuation of highly inflammable solvents, such as alcohols, which is risky at high temperatures $\left(260^{\circ} \mathrm{C}\right)$ and high pressures ( $\left.100 \mathrm{bars}\right)$. Hence, there is a need to produce silica aerogels using low-cost inorganic precursors such as sodium silicate (water glass) and drying at ambient pressure. In the present work, the silica aerogels have been synthesized using water glass as a precursor and drying the gels at atmospheric pressure. The effects of two different solvents (i.e., hexane and toluene) used for exchange on hydrophobicity and its physical properties of sodium-silica-based aerogels are discussed. So far, the aerogels produced using the sodium-silicate precursor and ambient pressure drying process are either opaque or translucent. This paper reports on the synthesis of transparent nanoporous silica aerogels using water glass as a precursor and followed by drying at atmospheric pressure.

\section{EXPERIMENTAL METHODS}

Referring to Figure 1, we used sodium silicate as a precursor solution (water glass, Shinwoo Materials Co. Ltd., South Korea) silica content $29 \%$ by weight (molar ratio $\mathrm{SiO}_{2}: \mathrm{Na}_{2} \mathrm{O}=3.02$ ). We prepared $100 \mathrm{ml}$ of $8 \%$ sodium silicate solution by weight by diluting $29 \%$ of sodium silicate solution with distilled water. Further, for replacing of $\mathrm{Na}^{+}$with $\mathrm{H}+$ ion, the solution was stirred for 30 minutes with an Amberlight ion exchange membrane (IR-120, pH 2.4, Merck, Germany). Before the ion exchange, the solution's pH was 12 and it was 2-3 after the stirring with the solution with Amberlight. The ion exchange is essential because the $\mathrm{Na}+$ ions, 
which are poorly soluble in alcohol and trapped in the gel network, cause a decrease in the dried gel's transparency. The hydrogel was prepared by adding $1 \mathrm{M} \mathrm{NH} \mathrm{NH}_{4} \mathrm{OH}$ in the silica solvent that was obtained by the ion exchange of sodium silicate. While stirring, the amount of $1 \mathrm{M} \mathrm{NH}_{4} \mathrm{OH}$ solution was adjusted until the $\mathrm{pH}$ of the solution became four. After the gelation, the hydrogels were aged for 12 hours in airtight containers (Rao et al, 2001).

In order to carry out the surface modification, the water present in the hydrogel was exchanged initially with ethanol. For this purpose, the gels were kept in $50 \mathrm{ml}$ of ethanol for 12 hours. After this step, to study the effects of hexane and toluene solvents, the gels were kept in the respective solvents at $40^{\circ} \mathrm{C}$ for 24 hours. The surface modification was then carried out by immersing the wet gels in baths containing various volumes (2 to $12 \mathrm{ml}$ ) of the trimethylchlorosilane (TMCS) in hexane and toluene for 24 hours. Finally, the gels were washed with $50 \mathrm{ml}$ of solvents in order to remove the unreacted trimethylchlorosilane (TMCS). While drying in an oven at different temperatures the gels were partly covered with aluminum foil perforated with small holes to control the evaporation of the solvent from the gels (Einarsrud and Nilsen, 1998). The gels were first dried at $60^{\circ} \mathrm{C}$ for 25 hours in an oven to determine the degree of spring-back effect of each type of the gel, and they were observed during drying. For complete removal of toluene and hexane solvents, gels were finally dried at $200^{\circ} \mathrm{C}$ for an hour.

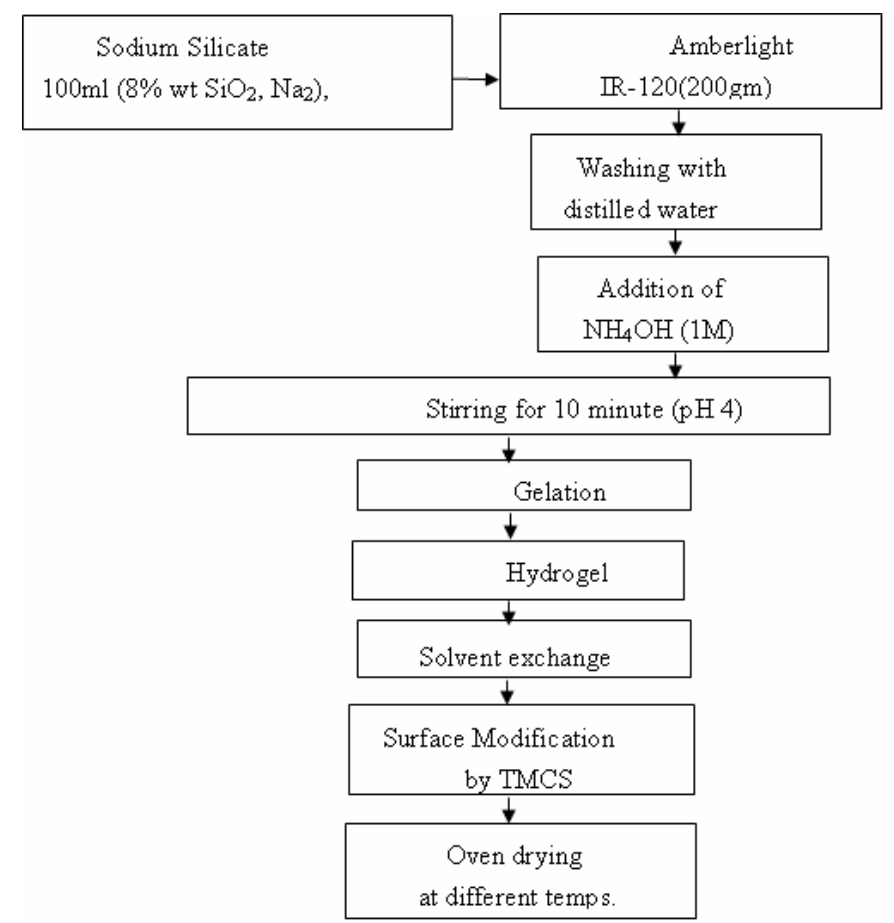

Figure 1. Experimental procedure for preparation of silica hydrogel and aerogel from sodium silicate solution. 
Hydrophobicity of the aerogels was tested by measuring the percentage of water absorbed by the aerogels by putting the aerogel samples directly on the water surface and observing the weight increase of the sample over time using a Dhona microbalance (Model: Dhona 100DS) with $10^{-5}$ gm accuracy. The percentage of optical transmittance of the modified aerogel (sample thickness of $1 \mathrm{~cm}$ ) was measured in the wavelength range of 300-800 nm using a UV-VIS recording spectrophotometer (UV-240 IPC, Shimadzu, Japan).

The pore size distributions (PSDs) were measured using a multiple-point nitrogen gas adsorption BET surface area analyzer (Model ASAP 2000) made by Micromeritics. A BET analysis from the amount of $\mathrm{N}_{2}$ gas adsorbed at various partial pressures (five points $0.05<p / p_{o}<0.3$, nitrogen molecular cross-sectional area $\left.=16.2 \AA^{2}\right)$ was used to determine the surface area, and a single condensation point $\left(p / p_{o}=0.99\right)$ was used to find the pore size and pore volume. Pore size distributions were calculated from the desorption isotherms (Wagh and Ingale, 1997).

In order to study thermal stability, hydrophobic aerogel samples weighing $10 \mathrm{mg}$ were heat-treated in an air medium from room temperature $\left(25^{\circ} \mathrm{C}\right)$ up to $450{ }^{\circ} \mathrm{C}$ with a controlled heating rate of $1.5^{\circ} \mathrm{C} \mathrm{min}{ }^{-1}$ using a microprocessor based Parr temperature controller (Model 4846) connected to a muffle furnace (A.H. Jeon Industrial Co. Ltd., Korea). Here, the thermal stability refers to the temperature up to which the aerogel retains its hydrophobicity (Rao and Haranath, 1999).

The surface modification was confirmed using infra-red (IR) spectroscopy using a Perkin-Elmer (Model No. 783) IR spectrophotometer. The three-dimensional Si-O-Si network microstructure of aerogels was observed using a scanning electron microscopy (SEM) analyzer (Model Philips XL-30).

\section{RESULTS AND DISCUSSION}

\section{EFFECT OF PH ON GELATION}

The preparation of hydrogels involves sol-to-gel transition. This transition is referred as a gelation where the sol becomes highly viscous and ceases to move even if the beaker is tilted or inverted (Scherer, 1989). The gelation time depends strongly on the amount of the catalyst added to the sol for gelation, as shown in Figure 2. This data can be explained using the general theory of silica polymerization. The condensation polymerization shows that silica gelation in aqueous solution involves an ionic mechanism. As the $\mathrm{pH}$ of the sol increased from one to five, the gelation time decreased from 600 to 30 minutes (Fig. 2). This is because an increase in the $\mathrm{pH}$ of the sol increases the rates of condensation reactions and, as a result, silica clusters aggregate at a relatively faster rate to form a three-dimensional, porous silica network in a short time (O'Connor and Sexton, 2003). The isoelectric point of an aqueous solution is found to be at $\mathrm{pH}=2$. Above $\mathrm{pH} 2$, the concentration rate is proportional to an $\mathrm{HO}^{-}$ion concentration and below $\mathrm{pH} 2$, to an $\mathrm{H}^{+}$ion concentration. 
At low pH range, silica particles have very little ionic charge. Consequently, they first aggregate into chains and finally into a three-dimensional gel network. For example, below $\mathrm{pH}$ 2, silica particles are positively charged; hence, they repel each other. So sols with the longest gelation time have a maximum temporary stability at about $\mathrm{pH}$ 1-2. Above $\mathrm{pH}$ 5-6 and up to eight, where gelation of silica sols begins, silica sols are negatively charged and, therefore, repel each other. Due to this fact, the gelation time at this range of $\mathrm{pH}$ increases. At around pH 5, silica monomer is converted into particles, which simultaneously aggregate into the gel network. Around this $\mathrm{pH}$, is the "gel point" where the viscosity begins to increase somewhat rapidly and gelation occurs. Above $\mathrm{pH}$ 5, silica particles are charged by ionization; hence, aggregation of silica sol particles is reduced.

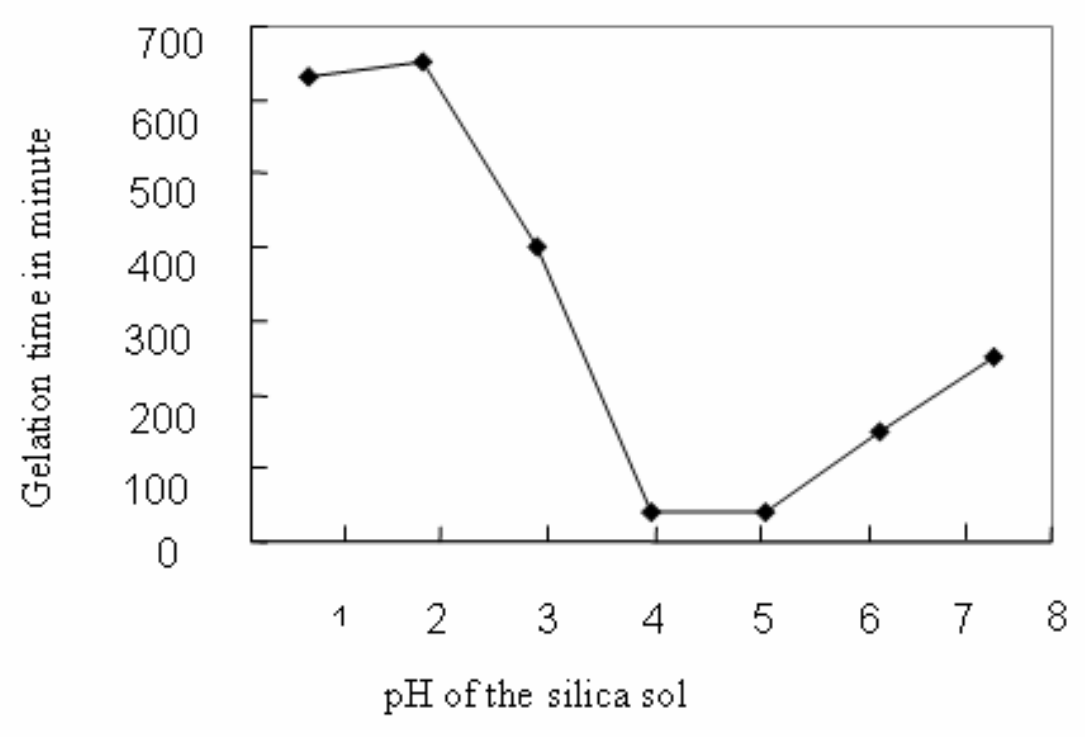

Figure 2. Effect of $\mathrm{pH}$ on hydrogel gelation.

\section{EFFECT OF SOLVENTS ON HYDROPHOBICITY AND OPTICAL TRANSMITTANCE OF DRIED SILICA AEROGELS}

Figure 3 shows the FTIR spectra of TMCS modified silica aerogels of different solvent exchanges. As referred in the handbook of infrared spectra (Pouchert , 1981) the broad absorption band at around 3,500 $\mathrm{cm}^{-1}$ is due to $\mathrm{OH}$ and other broad absorption bands between 1,050 and $1,200 \mathrm{~cm}^{-1}$, and at around $700 \mathrm{~cm}^{-}$ ${ }^{1}$, are due to Si-O-Si asymmetric stretching, symmetric stretching, and bending vibrations, respectively. The broad absorption peak at $1,100 \mathrm{~cm}^{-1}$ in the both the FTIR spectra corresponds to the Si-O-Si bonding which confirms the three dimensional Si-O-Si linking in the aerogels (Webb et al., 1997). The peak at $1,630 \mathrm{~cm}^{-1}$ represents the $\mathrm{Si}-\mathrm{OH}$ stretch vibrations. The $\mathrm{OH}$ and $\mathrm{Si}-\mathrm{OH}$ peaks are quite visible for hydrophilic silica aerogels. As shown in Figure 3, the less-stretched peak of $\mathrm{OH}$ bond appeared at 3,500 $\mathrm{cm}^{-1}$, indicating that the aerogels prepared using hexane solvent exchange are more hydrophobic than that 
of toluene solvent exchange. The hydrophobicity of the silica aerogels was tested by putting aerogel samples directly on a water surface. During the solvent exchange process it was observed that TMCS in toluene solvent is slightly less-reactive with the surface of $\mathrm{OH}$ group than in hexane solvent. This was due to differences in polarity of hexane and toluene solvents. Hence, the aerogels prepared through toluene solvent exchange are less hydrophobic than aerogels prepared using hexane solvent exchange. The surface TMCS reacts with the water and the hydroxyl groups on the surface and the surface could be represented by:
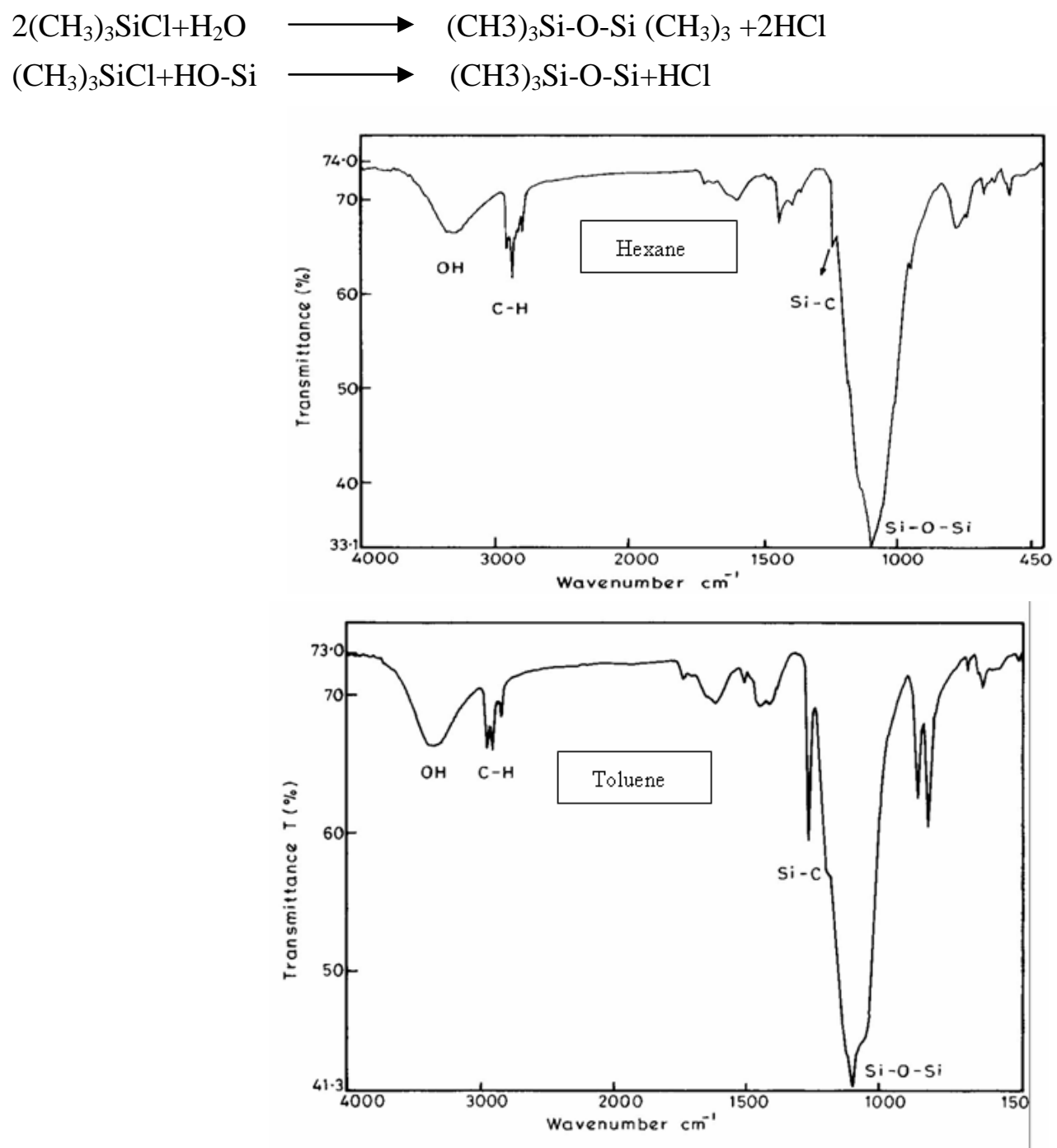

Figure 3. FTIR spectra of surface-modified, sodium-silicate-based aerogel with TMCS in hexane and toluene solvent. 
(a)

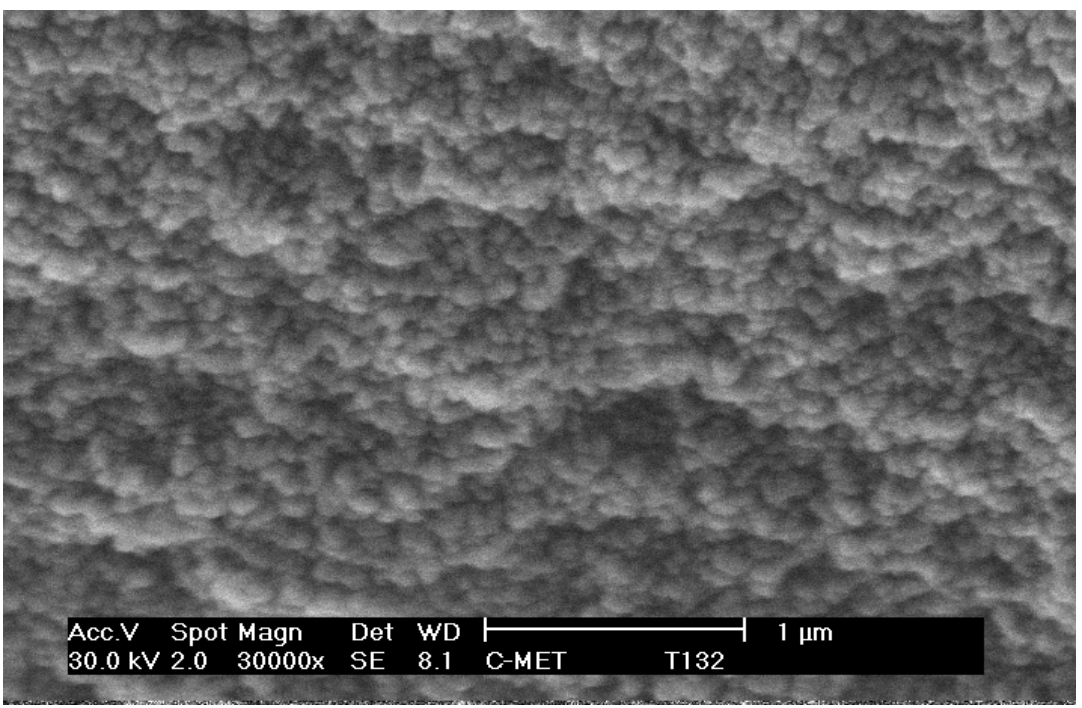

(b)

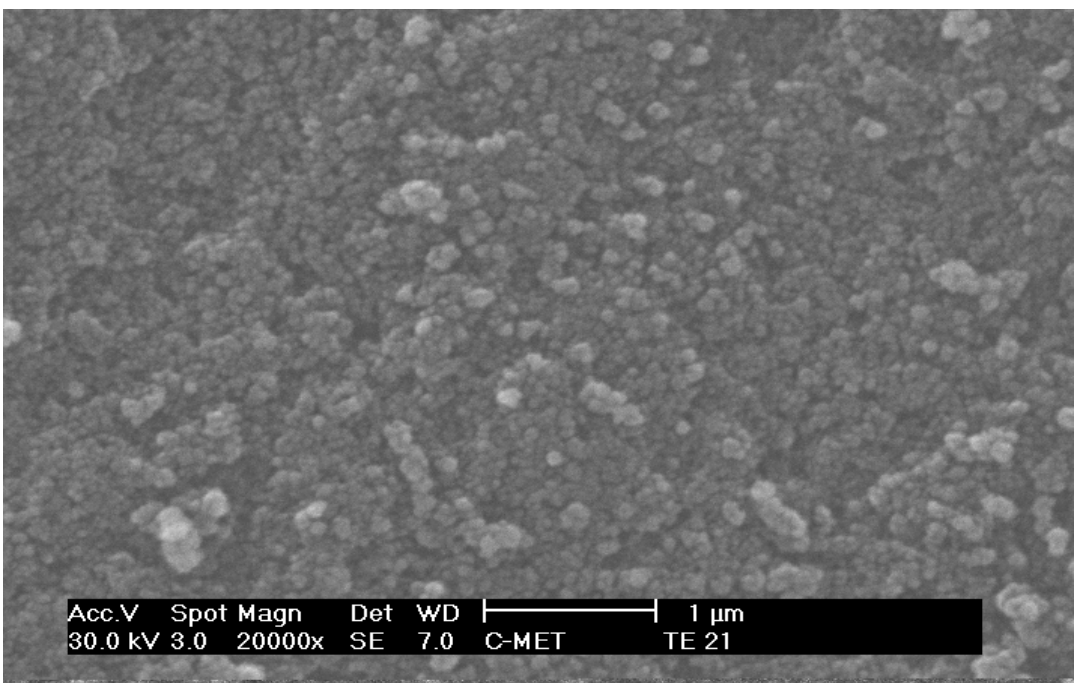

Figure 4. SEM microstructure of aerogel samples prepared using different solvents: (a) Hexane (8\% TMCS) (b) Toluene (8\% TMCS).

Figure 4(a) and (b), shows the SEM of the three-dimensional Si-O-Si microstructure of (8\% TMCS) of the aerogels. It is clear that hexane-modified aerogels have a compact microstructure with smaller clusters, but pores with non-uniform sizes; whereas, bigger and round-shaped clusters with an almost uniform pore size are observable in the SEM of aerogels prepared with toluene solvent exchange. The microstructure of hexane is seen to be very porous and loosely packed with smaller clusters

The surface area, pore size and optical transmittance of the silica aerogels synthesized via an ambientpressure drying process varied with the different preparative parameters, such as percentages of TMCS 
solutions, types of the solvent and aging time. The optical transmittance of the obtained silica aerogels are strongly affected by the volume percentages of TMCS solution. As shown in Fig. 5, as the volume of the TMCS solution increases from 2 to $12 \mathrm{ml}$, there is a decrease in the optical transmission of the synthesized silica aerogel. This is due to the more loosely bound, non-uniform cluster of $\mathrm{H}$ atoms being replaced by a methyl group $\left(-\mathrm{CH}_{3}\right)$ from the hydrolysable surface $\mathrm{OH}$ group of silica aerogel.

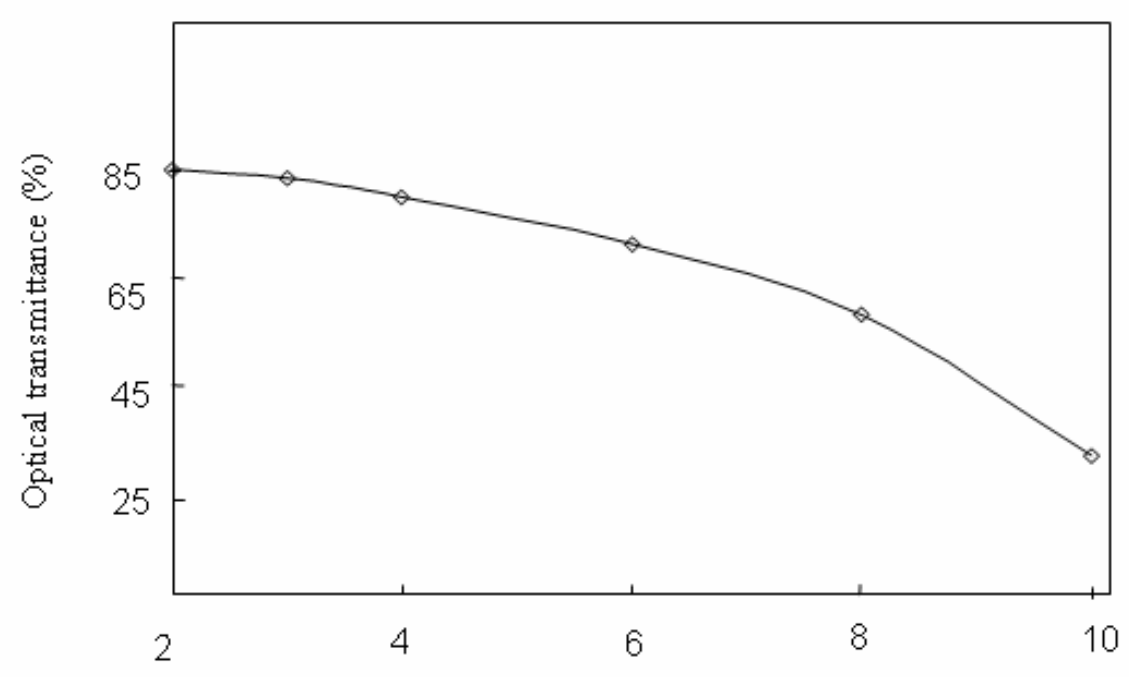

Volume \% of TMCS

Figure 5. Effect of volume \% of TMCS on transparency (at $700 \mathrm{~nm}$ ).

\section{EFFECT OF SOLVENTS ON BET SURFACE AREA AND PORE SIZE DISTRIBUTIONS}

The variation of the surface area with the percentages of the TMCS solution in hexane and toluene is shown in Figure 6(a) and (b), respectively. The surface area gradually increases as the percentage of the TMCS solution increases and depends on the type of solution. The surface area increased from 431-681 $\mathrm{m}^{2} / \mathrm{g}$ for hexane solvent exchange as percentages of TMCS increased and, in the case of toluene, it varied from $240-557 \mathrm{~m}^{2} / \mathrm{g}$. Figure 7 shows the effect of the gelling on the surface area and pore diameter of the aerogels for hexane and toluene solvents. The surface area varies from 681 to 431 $\mathrm{m}^{2} / \mathrm{g}$ for the hexane solvent exchange, and varies in the toluene solvent exchange from 596 to $227 \mathrm{~m}^{2} / \mathrm{g}$ with increase in aging time from 1 to 40 hours and figure 7(b) shows the aging time dependence of the average pore diameter. For the hexane solvent exchange, the pore diameter gradually increased from 2 to $26 \mathrm{~nm}$ under the acidic condition (pH3.7) and in the case of the toluene solvent exchange, it rapidly increased from 2 to $13 \mathrm{~nm}$. The increased in pore diameter is caused due to aging, smaller pores dissolved in to larger pores. From this result, it is clear that the condensation polymerization of silanol 
groups in the silica is more effective than the acidic condition.
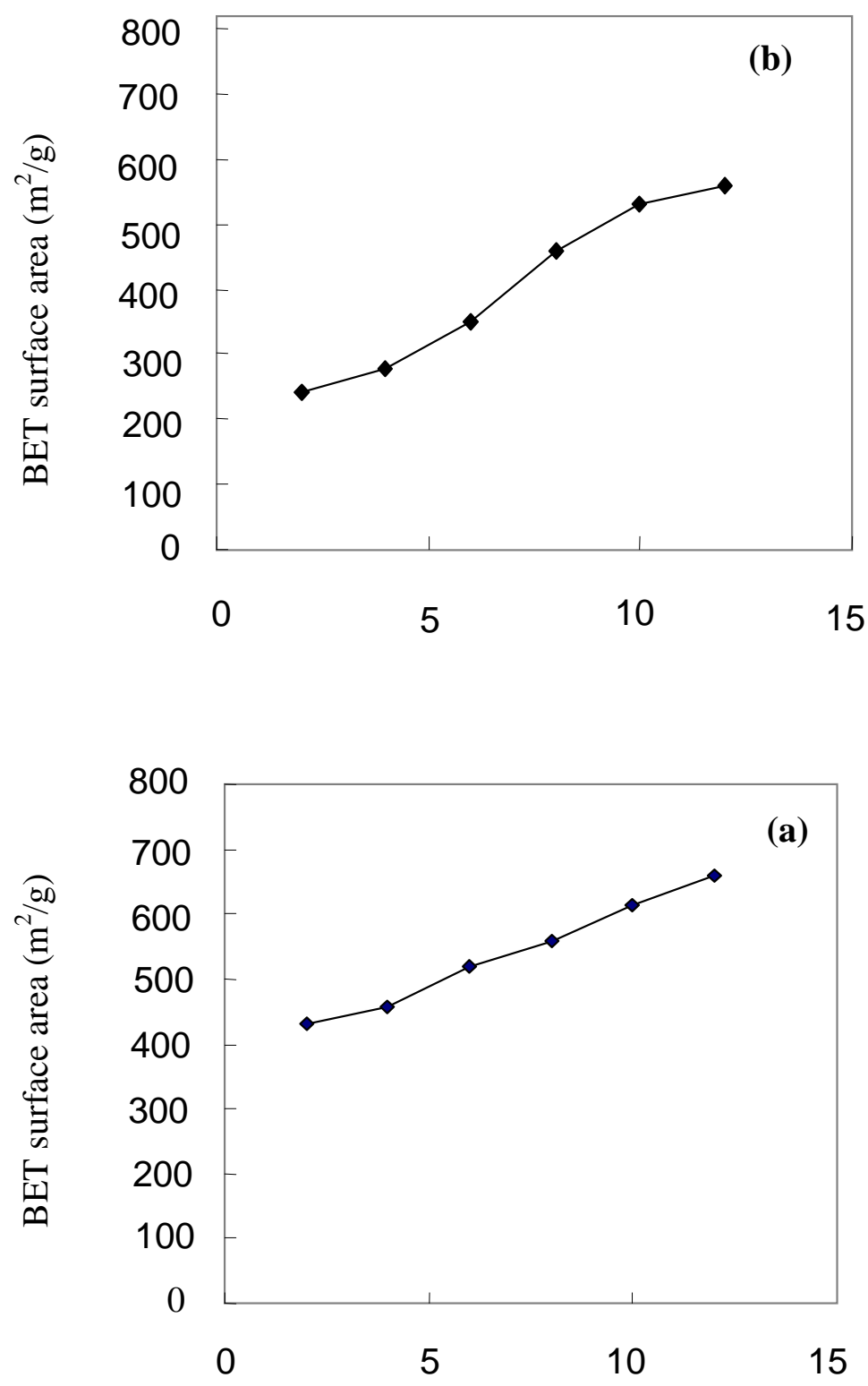

(a) \% volume of TMCS

Figure 6. Variation of BET surface area with \% of TMCS in (a) hexane and (b) toluene solvent, respectively. 
(a)

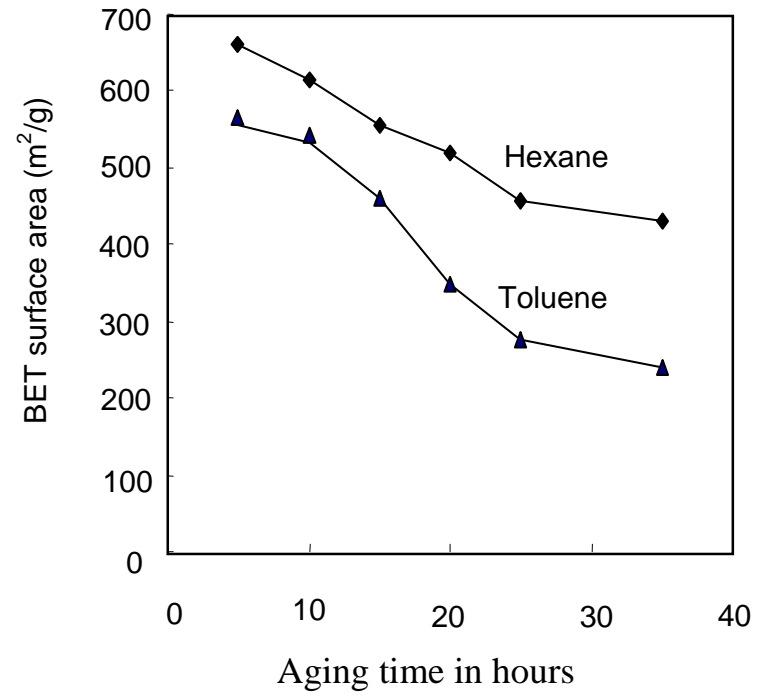

(b)

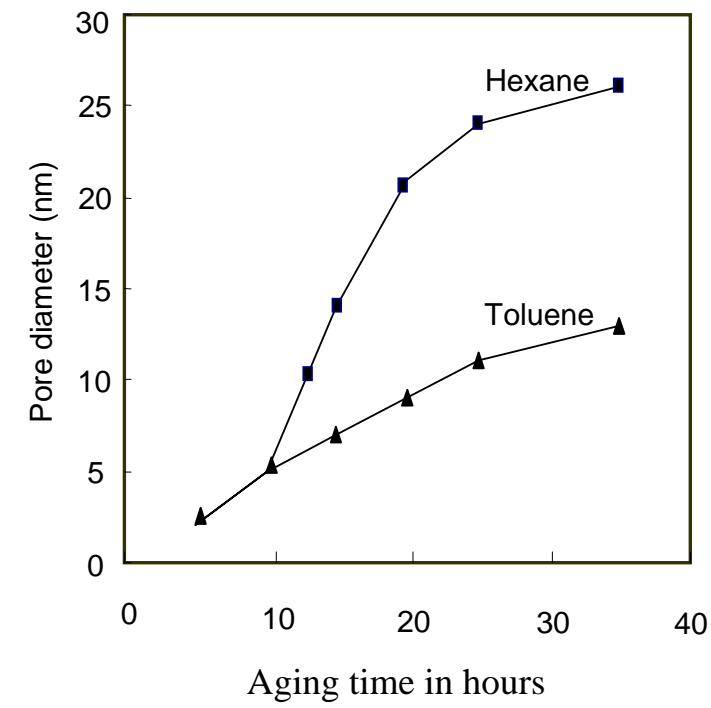

Figure 7. Variation of the (a) BET surface area and (b) pore diameter with aging time at acidic condition ( $\mathrm{pH}$ 3.7), hexane and toluene solvent exchange respectively.

Figure 8 shows the BET result of the pore size distribution of silica aerogel obtained from hexane and toluene solvent exchange .The average pore diameter obtained from hexane solvent exchange is $10 \mathrm{~nm}$, and for the toluene solvent exchange, it was $20 \mathrm{~nm}$. The enlarging of the pore diameters is considered to be due to the dissolution of smaller pores and the condensation polymerization of silanol groups .The gels were aged at $40^{\circ} \mathrm{C}$ in an oven for $1-24$ hours. The pore diameter decreased with gel aging. This effect is due to the fact that with increased aging time, the shrinkage volume of the aerogel increases. During the gel aging, a number of physical and chemical changes occur, such as condensation of $\mathrm{Si}-\mathrm{OH}$ groups, syneresis, coercing, and segregation, cause decrease in pore diameter of silica aerogel.

\section{CONCLUSIONS}

Silica aerogels were obtained by ambient pressure drying with systematic variation of different parameters, such as varying the percentages of TMCS in hexane and toluene by the derivatization method. It has been found that the silica aerogel obtained at ambient pressure through solvent exchange was strongly affected by types of the solvent on its physical properties, such as surface area, pore diameter and hydrophobicity. The surface area of the obtained silica aerogels varied from 431 to $681 \mathrm{~m}^{2} / \mathrm{g}$ for hexane solvent exchange with different percentages of TMCS solution, and in the case of toluene solvent with different percentages of TMCS solution, it varied from 240 to $557 \mathrm{~m}^{2} / \mathrm{g}$. It was observed that the 
surface areas of silica aerogels increased with percentages of the TMCS solution in toluene or hexane solvent. The best-quality, water-glass-based silica aerogels, in terms of high surface area $\left(681 \mathrm{~cm}^{2} / \mathrm{g}\right)$, more hydrophobic, low pore diameter $(10 \mathrm{~nm})$, and high optical transparency were obtained with $50 \mathrm{ml}$ of the hexane solvent in an 8\% TMCS solution.
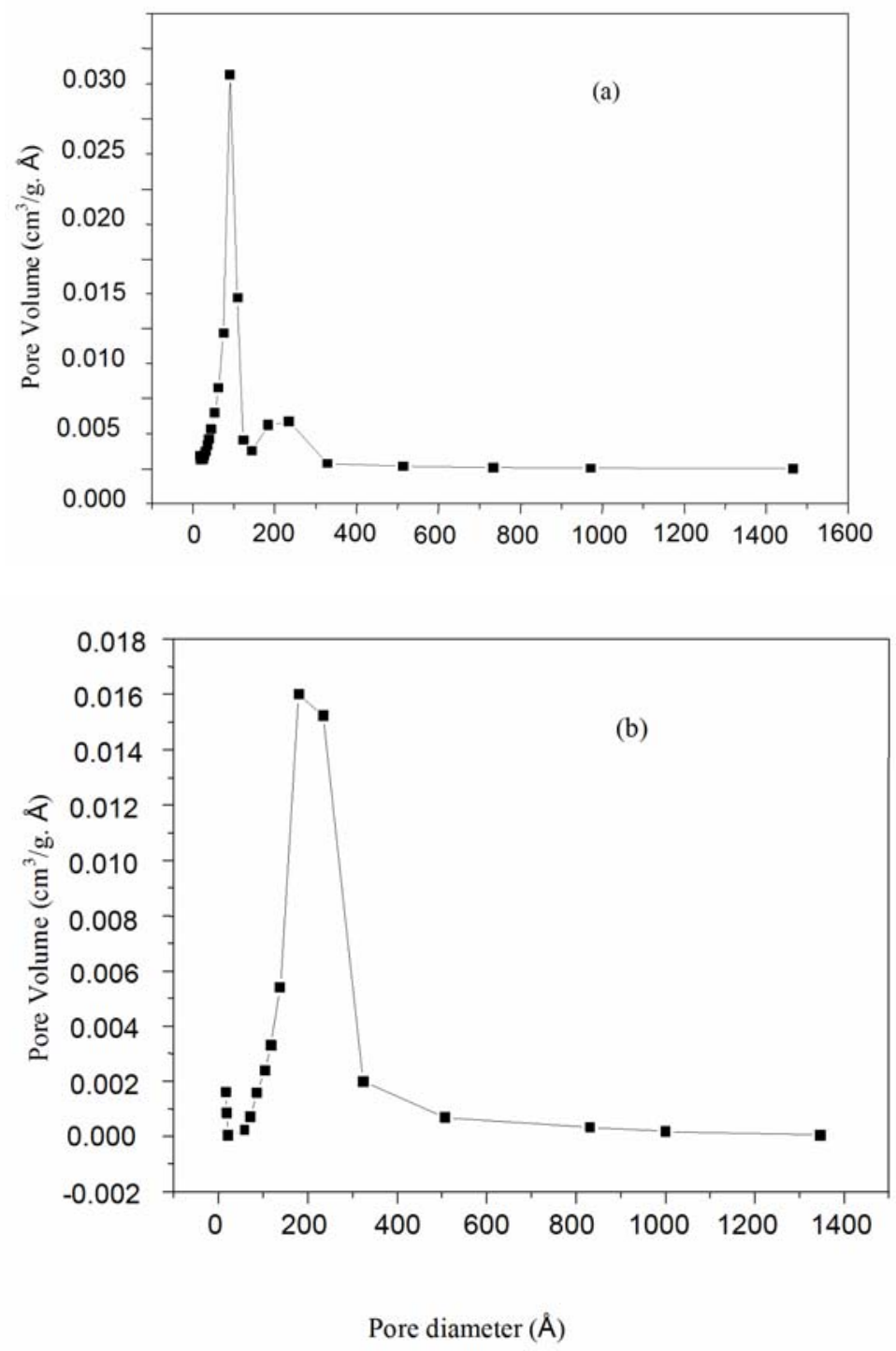

Figure 8. Pore size distribution determined for the water-glass-based aerogels of (a) hexane and (b) toluene solvent exchanged with $8 \%$ of TMCS solution. 


\section{ACKNOWLEDGEMENTS}

The authors gratefully acknowledged financial support received from the government of Korea under the research project on "Silica Aerogels." We also sincerely thank the reviewers for their valuable suggestions and corrections which have considerably improved the quality of the manuscript.

\section{REFERENCES}

Ashley, C.S., Reed, S.T., Brinker, C.J., Walko, R.J., Ellefson, R.E., Gill, J.T. (1992), Chemical Processing of Advanced Materials. L.L. Hench and J.K. West (Eds.), Wiley, New York, p. 989.

Haranath, D., Wagh, P.B., Pajonk, G.M., and Rao, A.V. (1997), Influence of Sol-Gel Processing Parameters on the Ultrasonic Sound Velocities in Silica Aerogels. Materials Res. Bull. 32:1079-1089.

Hrubesh, L. W. (1988), Aerogel Applications. J. Non-Cryst. Solids 225:335-342.

Chunha, J.P., Neves, F., Lopes, M.I. (2000), On the Reconstruction of Cherenkov Rings from Aerogel Radiators. Nucl. Instrum. Meth. A452:401-421.

Deng, Z., Wang, J., Wu, A., Shen, J., Zhou, B. (1998), High Strength $\mathrm{SiO}_{2}$ Aerogel Insulation. J. NonCryst. Solids 225:101-104.

Einarsrud, M.-A. and Nilsen, E. (1998), Strengthening of Water Glass and Colloidal Sol-Based Gels by Aging in TEOS. J. Non-Cryst. Solids 226:122-128.

O’Connor, D.J. and Sexton, B.A. (2003), Surface Analysis Method in Materials Science, R. St. C. Smart. (Eds.), Springer. Http://www.springer.de/physics/

Pajonk, G. M. (1991), Aerogel Catalysts. Appl. Catal. 72:217-266.

Pajonk, G. M., Teichner, S.J. (1985), Proceedings of the First International Symposium on Aerogels. J. Fricke (Ed.), Springer Press, Berlin, pp. 73-89.

Pouchert, C.J. (1981), The Aldrich Library of Infrared Spectra, $3^{\text {rd }}$ Edition, Aldrich Chemistry Co., wisconisanoo, WI.

Prakash, S.S., Brinker, C.J., Hurd, A. J., Rao, S. M. (1995), Silica Aerogel Films Prepared at Ambient Pressure by Using Surface Derivatization to Induce Reversible Drying Shrinkage. Nature 374: 439-443.

Prakash, S.S., Brinker, C.J., Hurd, A. J. (1995), Silica Aerogel Films at Ambient Pressure. J. Non-Cryst. Solids 190:264-275.

Rao, A.V. and. Haranath, D. (1999), Effect of Methyltrimethoxysilane as a Synthesis Component on the Hydrophobicity and Some Physical Properties of Silica Aerogels. Microporous and Mesoporous Materials 30:267-273.

Rao, A.V., Nilsen, E., and Einarsrud, M.-A. (2001), Effect of Precursors, Methyalation Agents and Solvents on the Physicochemical Properties of Silica Aerogels Prepared by Atmospheric Pressure Drying Method. J. Non.-Cryst. Solids 296:165-171. 
Scherer G.W. (1989) J. Non-Cryst. Solids 100:77-92.

Schmidt, M. and Schwertfeger, F. (1998), Applications for Silica Aerogels Products. J. Non-Cryst. Solids 225: 364-368.

Schwertfeger, F., Frank, D., Schmidt, M. (1998), Hydrophobic Water-Glass-Based Aerogels WithoutSolvent Solvent Exchange or Supercritical Drying. J. Non-Cryst. Solids 225:24-29.

Schwertfeger, F., Frank, D., Schmidt, M. (1998), Hydrophobic Water-Glass-Based Aerogels WithoutSolvent Solvent Exchange or Supercritical Drying. J. Non-Cryst. Solids 225:24-29.

Svendsen, S. (1992), Solar Collectors with Monolithic Silica Aerogel. J. Non-Cryst. Solids 145:240-243. Yang, H.S., Choi, S.Y., Hyun, S.H., Park, H. H., Hong, J. K. (1997), Ambient-dried Low Dielectric $\mathrm{SiO}_{2}$ Aerogel Thin Film, J. Non-Cryst. Solids 221:151-156.

Wagh, P. B., Ingale, S.V. (1997), Influence of Temperature on the Physical Properties of TEOS Silica Aerogels. Ceramics International 28:43-50.

Webb, P.A., Orr, C., Camp, R.W., Olivier, J.P., Yunes, Y.S. (1997), Analytical Method in Fine Particle Technology, Micromeritics Instrument Corporation, Norcross, GA USA, pp. 53-87.

Received for review, January 17, 2006 Accepted, March 26, 2006 